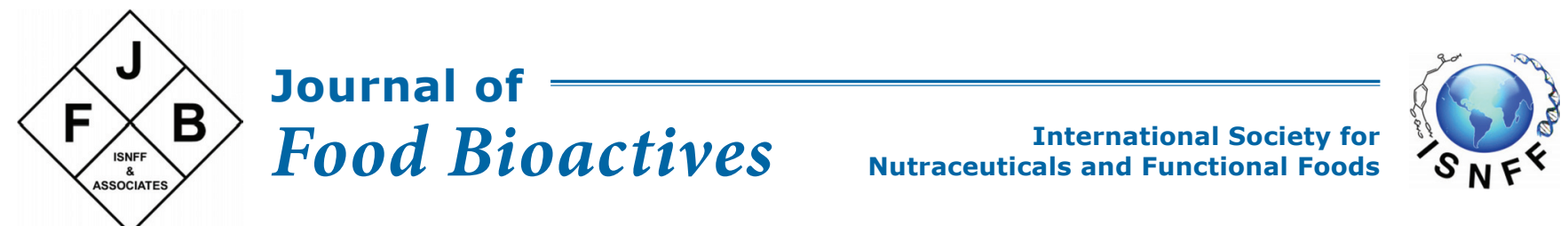

\title{
Do traditional food cultures play a role in COVID-19 for health and immunity?
}

\author{
Fereidoon Shahidi
}

Department of Biochemistry, Memorial University of Newfoundland, St. John's, NL, Canada A1B 3X9. E-mail: fshahidi@mun.ca DOI: $10.31665 /$ JFB.2021.14274

Received: June 30, 2021; Revised received \& accepted: June 30, 2021

Citation: Shahidi, F. (2021). Do traditional food cultures play a role in COVID-19 for health and immunity? J. Food Bioact. 14: 1-3.

\begin{abstract}
The goal of this contribution is to provide a summary report on the Scientific Roundtable of the International Union of Food Science and Technology (IUFoST) about the role of traditional food cultures on health promotion and immunity in the COVID-19 pandemic era. The role of food bioactive ingredients and effects on food safety and security as well as sustainability were discussed by the panel members and recommendations were made to take advantage of the historical evidence for providing scientific basis for the efficacy of the ingredients and their globalization.
\end{abstract}

Keywords: COVID-19; Traditional foods; Health; Immunity; Traditional food cultures.

This brief write-up provides summary of the Scientific Roundtable \#8 under the auspices of the International Union of Food Science and Technology (IUFoST) held on April 29, 2021. The meeting was chaired by Dr. Vish Prakash, Dr. Pingfan Rao and Dr. Fereidoon Shahidi with Dr. Hongda Chen and Dr. Stephane Guilbert as reporters and facilitators. The panel members provided views about the role of traditional food cultures in health promotion as affected by COVID-19 pandemic era.

The traditional and ethnic foods play an increasing role in today's multi-cultural societies. The wisdom, traditional practices, and the preservation of foods through the knowledge of food culture and civilization combined with today's food science expertise provide a unique opportunity to understand the benefits of these foods better than ever before. The advances of food engineering have helped in scale up and preservation techniques retaining the texture, flavour and the overall sensory qualities of processed traditional Foods available in the market. The modern tools of food science and food composition and our better understanding of bioactive compounds responsible for health promotion and disease risk reduction have helped in better and convenient processing of this class of foods with value addition to the raw material with the mix of food culture and traditional food habits. These aspects provided the focus of the roundtable reported here. In fact, many of the traditional foods and remedies were in place for decades and centuries without the exact scientific articulation to back up their efficacy and mechanism of action beyond anecdotal evidence.
African Food Prize Laureate Professor Ruth Oniang'o underlined the great diversity of African traditional foods and food cultures. She put forward African specificities that are very current in the evolutions and needs of the food systems. For example, the alternative sources of proteins, vegetable proteins, fermented foods, insects, such as locusts were noted. She indicated that the traditional African foods are usually colourful and spicy, not just for flavour but also as a preservation method. She specified that food is not just for nutrition but a means for building friendship and with social impacts. Dr. Oniang'o emphasized the role of traditional food cultures for their contribution in micronutrients and the strengthening of immune defenses.

The South American perspective was offered by Professor Jaime Amaya-Farfan who pointed out the hybridization of food cultures between indigenous cultures and European, African, and Asian cultures imported by immigrants. He also showed the importance of the adaptation of these different food cultures to the local food resources, for example the substitution of cereals by pseudo-cereals such as quinoa and amaranth or the use of the very important resources of the Amazon or the consumption of nutritional foods rich in bioactive phytochemicals, especially fruits, some of which have remained unutilized. The variation of foods is quite tangible among different countries in the region.

Dr. Hiroya Kawasaki of Ajinomoto Company highlighted the specificities of Japanese cuisine, in particular the use of seasonal products but above all the use of dashi-based products, the plat- 
form broth of Japanese cuisine, which provides umami flavour (MSG-type) and the aromas generated upon Maillard reaction that makes it possible to prepare products with a low-fat and low calorie with mild flavour that are generally acceptable by all and provide important bioactive compounds.

Professor Lara Hanna-Wakim highlighted the health and environmental benefits of the Mediterranean diet, which is based on a high consumption of fruit and vegetables, a moderate consumption of fish and dairy products and olive oil but a low amount of red meat. She also highlighted that the Mediterranean diet is not just a mere collection of specific foods but is a way to promote social interaction and increase sociocultural values. She indicated that these foods have the benefit of improving heart, brain, and bone health, as well as controlling blood sugar and weight through their antioxidant effects rendered by the presence of bioactive components. She added that the COVID-19 pandemic is associated with various clinical, mental, and psychological complications that has challenged healthcare and social systems. She added that obesity, central fat distribution and adiposity-associated chronic diseases may lead to poor COVID-19 outcomes. Indeed, obese COVID-19 patients were found to develop more serious complications than others.

Prof. Rekha Singhal focused on the great diversity of the Indian food cultures and India's unique positioning in traditional foods for over 5,000 years. She mentioned how these traditional foods show their efficacy both as a way of preserving food and also as helpful medicinal benefits, especially spices, on cardiovascular disease, inflammation, diabetes, and Alzheimer. The wisdom in the intelligent and optimal use of resources for immune boosting and inclusion of pro-, pre- and symbiotic ingredients was also mentioned. She demonstrated the many benefits of herbs and spices, millets, legumes, pulses, and nutra-grains especially for immunity boosting or fighting against diseases of modern times with combinatorial effects. The importance of Ayurvedic style cooking was also emphasized. The concept of tadka or tempering to improve bioavailability and abundant use of ghee and other specialty oils with known bioactivities was noted.

Dr. Lijing Ke presented in a most original way how food complies with the Chinese traditional philosophy of yin (cold) and yang (hot). He related Yin to pacifying food (ingredients) such as tea, bitter, sour, sweet and mouth-watering with anti-inflammatory effect while Yang to spicy, salty and MSG type food (ingredients) with consequences of thirst and inflammatory effects. He also showed how food nanoparticles in soups and infusions are vehicles for bioactive compounds and micronutrients. Furthermore, the role of food at a molecular level to bring about metabolic changes in such small amounts is being understood with models and the interactions at cellular level.

Professor Cherl-Ho Lee, the founder of the Korea Food Security Research Foundation, and author of "A History of Korean Food, Korean Foodways from Prehistory to the Present" provided a basis for the origin of cooking methods and fermented foods, and for future contributions of Korean foodways to the world, in particular with regard to the intersection of food and traditional Eastern medicine. He noted that "A survey of classical literature [in Korea] reveals the historical background and manufacture methods of traditional food categories, such as rice cakes, Korean sweets, fermented sauces...". He related some of the traditional food preparations to the existing pottery in Korea, including kimchi, joetkal and rice wine.

The roundtable noted the following:

1. The importance of diversity of food and materials across the world needs to be enhanced to preserve global biodiversity particularly in light of the natural environment under threat through a variety of external condition including climate change as well as health crises such as COVID-19. The preservation of natural resources of food and material is critical for the future.

2. Scientific methodologies need to strengthen the connection between food and health. For example - using nanoscale science to look at the importance of micro-/nano-structural changes of food materials in addition to compositions in human GI tract. It is inconceivable in today's scientific environment that the micro/nano structure of a food component would not have an effect on the health outcome and efficacy of a food ingredient, like that shown in drug delivery research.

3. Acknowledgement of the perceptions of food as a part of culture needs be strengthened to enhance an effective approach. Food is part of culture. Food is not food alone because it involves peoples' philosophical views as a part of their lives.

This scientific roundtable underlined the great diversity of traditional foods and food cultures. What appears very important is that specificities of traditional foods are very relevant to the evolutions and needs of the food systems regarding the actual challenges on sustainability, nutrition, and health. Thus, efficiency and wisdom in the intelligent and optimal use of resources, the promotion of proteins with a low environmental impact such as vegetable proteins, fermented foods, insects and the many benefits of traditional foods, herbs, and spices, especially in strengthening immunity or fighting modern day diseases. Scientific documentation and the traditional wisdom of Food Culture needs to be brought together and shared with the medical profession, nutritionists, sociologists, and other scientists as well as the consumers.

To achieve these, it is essential to proceed with the organization of a Global Database through IUFoST. One of the ways food science and technology can contribute to protection and sustainability of traditional foods and processes is to be able to better quantify fundamental qualities of traditional foods. Therefore, IUFoST can promote the collection of databases and the research on standardized effectiveness indicators or methods to characterize environmental impact and major health and nutritional benefits. Traditional spices and herbs have been used from time immemorial to promote good health and as medicine and they are being acknowledged for their immune-boosting function. Many recent scientific reports support this and a new emerging area in this respect is very important to nurture.

Traditional foods of some regions remain unknown as potential food sources in other parts of the world. Through capacity building, such products can be introduced in future food systems into new markets. There are opportunities available for innovation through an understanding of traditional raw materials, ingredients, and processes. Industries can learn more about market needs around consumer preferences for healthy, environmentally acceptable, and socially friendly food products that are available economically, provide local employment, and through local sources that help to promote a healthy diet. Consumer preference can be guided through education by the lessons of the traditional food products, processes that provide safety and sustainability for the food systems.

There is a critical need for women to be educated in Food Science and Technology as they are drivers in agriculture, traditional food production and processing in many parts of the world. An overall improvement in STEM learning for women will serve an improved foundation for them to engage in learning, practicing, and contributing to food science and technology.

Research, pilot plants, funding need to be made available to food scientists, technologists, and engineers because of their cen- 
tral role in a sustainable and resilient food system. Public and private investment in food science research will accelerate the discovery of health benefits of traditional foods precisely applicable to individual consumers or subsects of the population with specific health issues. It will fill the knowledge gaps important for developing and tailoring new food products. Technology developments will strengthen the sustainability and resilience of future food supply systems.

Traditional foods contain principal technological routes to reduced energy utilization in processing: natural preservation; retention of micro-nutrients; and a wide variety of acceptable tastes and textures and most importantly the resilience. All of these are technologies transferable into new product forms anywhere. As such they represent the basis of sustainable innovation. The regulatory and consumer concerns associated with high tech novel processing vis a vis the same issues with traditional processing would be a good synergy to make new and innovative products.

It is recommended and expected that experts representing food science and technology in the areas of fortification, food safety, food processing and product development, production and market economy, human nutrition, food engineering, and quality control to work with partners in agriculture, animal breeding, and genetics, livestock processing, social agencies, government regulatory bodies, and UN agencies, including CODEX. Capacity building and collaborative research efforts are required from governments and must include industry engagement; overall a cross-discipline engagement is needed with food science and technology as the coordinating component to ensure the safety and sustainability of the traditional food chain. To learn from countries with historical lineage nearly 5,000 years it is important to understand every aspect of its emergence and sustainability over 200 generations with resilience built is an important and remarkable lesson the modern food scientists have to learn from this very vast knowledge.

To upgrade locally produced traditional foods and allow research on all aspects of food production from farmer to consumer, food pilot plants are a necessity in the chain if an impact is expected. This need is particularly urgent in many parts of the world, especially Africa and Asia to learn from this. Traditional food systems have many ingredients and processes that require understanding of how it was standardized and the research and technology to ensure food safety as many the steps involved might perhaps been missed over many centuries or the documentation is missing in some countries. India and China are an exception with lots of documentation dating back to 2,000 to 3,000 years. These pilot plants and industry involvement are a necessity at the local and regional levels and beyond for its large-scale production and distribution of traditional foods not only provide a distilled wisdom of each region's food culture and tradition but also offer a treasure of knowledge to mine by scientists, technologists, and engineers for the benefit of all human beings. 